\title{
Pacific
}

Journal of

Mathematics

\section{A GENERALIZATION OF MAXIMAL FUNCTIONS ON COMPACT SEMISIMPLE LIE GROUPS}

\author{
HENDRA GUNAWAN
}




\title{
A GENERALIZATION OF MAXIMAL FUNCTIONS ON COMPACT SEMISIMPLE LIE GROUPS
}

\author{
HendRa Gunawan
}

\begin{abstract}
Let $G$ be a compact semisimple Lie group with finite centre. For each positive number $s$, let $\mu_{s H}$ denote the $\operatorname{Ad}(G)$-invariant probability measure carried on the conjugacy class of $\exp (s H)$ in $G$. With this one-parameter family of measures, we define the maximal operator $\mathscr{M}_{H}$ on $\mathscr{C}(G)$. We then estimate the Fourier transform of $\mu_{s H}$ and of some derived distributions. Our result leads to the boundedness of $\mathscr{M}_{H}$ on $L^{p}(G)$, for all $p$ greater than some index $p_{0}$ in $(1,2)$. This generalizes a recent result of $M$. Cowling and $\mathbf{C}$. Meaney [2].
\end{abstract}

Introduction. Let $G$ be a compact semisimple Lie group of rank $l$ with finite centre, and with its Haar measure normalized to have total mass 1 . Let $\mathfrak{g}$ denote its Lie algebra, and let $\mathfrak{h}$ be a maximal toral subalgebra of $\mathfrak{g}$. We denote by $\Phi$ the root system of $\left(\mathfrak{g}^{\mathbf{c}}, \mathfrak{h}^{\mathbf{c}}\right)$, and fix $\Delta=\left\{\alpha_{j}: j \in I\right\}$, where $I=\{1, \ldots, l\}$, to be a base of $\Phi$ (as in [3, $\S 10.1])$. With respect to $\Delta$, we write $\Phi^{+}$for the set of positive roots, whose members are of the form

$$
\alpha=\sum_{j \in I} n_{j}(\alpha) \alpha_{j}
$$

with $n_{j}(\alpha) \in \mathbf{Z}^{+} \cup\{0\}$ for all $j \in I$, and $\Lambda^{+}$for the set of dominant weights, which parametrizes the dual object of $G$.

We equip the Lie algebra $\mathfrak{g}$ with the positive definite inner product $(\cdot, \cdot)$ derived from the Killing form. For each $\nu \in \mathfrak{h}^{*}$, we define $H_{\nu} \in \mathfrak{h}$ by

$$
\nu(H)=\left(H_{\nu}, H\right) \quad \forall H \in \mathfrak{h} .
$$

We also transfer the inner product to $\mathfrak{h}^{*}$ via

$$
\left(\nu, \nu^{\prime}\right)=\left(H_{\nu}, H_{\nu^{\prime}}\right) \quad \forall \nu, \nu^{\prime} \in \mathfrak{h}^{*} .
$$

The norm on $\mathfrak{h}^{*}$ and $\mathfrak{h}$, induced by these inner products, will then be denoted by $|\cdot|$.

We choose a regular element $H \in \mathfrak{h}$, for which $\alpha(H) \neq 0$ for all $\alpha \in \Phi^{+}$, and fix $R>0$ such that $\exp (s H)$ is regular in $G$ for any 
$s \in(0, R)$. For a continuous function $f$ on $G$, the maximal function $\mathscr{M}_{H} f$ is defined by

$$
\mathscr{M}_{H} f(x)=\sup _{s \in(0, R)}\left|\mu_{s H} * f(x)\right| \quad \forall x \in G,
$$

where $\mu_{s H}$ is the $\operatorname{Ad}(G)$-invariant probability measure carried on the conjugacy class of $\exp (s H)$ in $G$. This definition generalizes one in the paper of Cowling and Meaney [2], in which $H$ was a particular regular element of $\mathfrak{h}$. Our main results are the following.

THEOREM A. For all $k=0,1,2, \ldots$, there exist positive constants $C_{k}=C_{k}(H)$ such that

$$
\left|\left(\frac{\partial}{\partial s}\right)^{k} \hat{\mu}_{s H}(\lambda)\right| \leq C_{k} \frac{(1+|\lambda|)^{k}}{(1+s|\lambda|)^{\gamma}} \quad \forall s \in(0, R), \lambda \in \Lambda^{+},
$$

where $\gamma=\min _{j \in I}\left|\left\{\alpha \in \Phi^{+}: n_{j}(\alpha) \geq 1\right\}\right|$.

It is clear that Theorem $\mathrm{A}$, together with the arguments of [2], imply the boundedness of $\mathscr{M}_{H}$ on $L^{p}(G)$ for all $p>1+(2 \gamma)^{-1}$. So we state

Theorem B. For all $p>1+(2 \gamma)^{-1}$, with $\gamma$ as above, there exist positive constants $C_{p}=C_{p}(H)$ such that

$$
\left\|\mathscr{M}_{H} f\right\|_{p} \leq C_{p}\|f\|_{p} \quad \forall f \in \mathscr{C}(G) .
$$

We prove Theorem A by handling first the case when $G$ is simple, and then extend the result to the semisimple case. Our method is based on arguments of representation theory, involving formulae for characters and dimensions, a study of root systems, the theory of weights, and properties of the Weyl group, all developed in the first part of this note. The proof of Theorem A will be given in the second part. It is clear that Theorem $\mathrm{A}$ is sharp since the explicit expression used in [2] for the particular case in which $H=H_{p}$ shows no improvement is possible. In the third part of this note, we give an example which shows that Theorem B too is sharp at least in the case where $G=\mathbf{S U}(2)$.

Some related results can be found in M. Christ [1] and C. D. Sogge and E. M. Stein [5].

Throughout this note, the expressions $C, C_{k}$, and $C_{k_{1}, k_{2}, k_{3}}$ denote various positive constants which possibly vary from line to line. These constants may depend on $G$, and some may also depend on the choice 
of $H$. When a constant, $C$ say, depends on $H$, we write $C(H)$ in place of $C$.

We are grateful to Professor M. Cowling for his valuable suggestions during the preparation and the writing of this note. In particular, we would like to thank him for helpful discussions concerning the sharpness of the $L^{p}$-estimate.

1. Representation theoretic arguments. We shall assume throughout this part that the Lie algebra $\mathfrak{g}$ is simple.

1.1. We start with some formulae for characters and dimensions of representations of $G$. To each $\lambda \in \Lambda^{+}$, we associate the representation $\pi_{\lambda}$, the set of weights $\varpi_{\lambda}$, the character $\chi_{\lambda}$, and the dimension $d_{\lambda}=\chi_{\lambda}(1)$. For all $\lambda \in \Lambda^{+}$, we have (see $\left.[3, \S 22]\right)$

$$
\chi_{\lambda}(\exp (H))=\sum_{\lambda^{\prime} \in \varpi_{\lambda}} m_{\lambda}\left(\lambda^{\prime}\right) \exp \left(i \lambda^{\prime}(H)\right),
$$

where $m_{\lambda}\left(\lambda^{\prime}\right) \in \mathbf{Z}^{+}$is the multiplicity of $\lambda^{\prime}$ in $\pi_{\lambda}$. Accordingly,

$$
d_{\lambda}=\sum_{\lambda^{\prime} \in \varpi_{\lambda}} m_{\lambda}\left(\lambda^{\prime}\right)
$$

Let $\mathscr{W}$ be the Weyl group of $\left(\mathfrak{g}^{\mathbf{c}}, \mathfrak{h}^{\mathbf{c}}\right)$, generated by the reflections $\sigma_{\alpha}$ corresponding to $\alpha \in \Delta$. Introduce the special element $\rho=\frac{1}{2} \sum_{\alpha \in \Phi^{+}} \alpha$. For all $\lambda \in \Lambda^{+}$, the character and dimension formulae of Weyl read (see [3, §24.3])

$$
\chi_{\lambda}(\exp (H))=\frac{\sum_{\sigma \in \mathscr{W}} \operatorname{det}(\sigma) \exp (i \sigma(\lambda+\rho)(H))}{\prod_{\alpha \in \Phi^{+}} 2 i \sin \frac{1}{2} \alpha(H)}
$$

and

$$
d_{\lambda}=\prod_{\alpha \in \Phi^{+}} \frac{(\lambda+\rho, \alpha)}{(\rho, \alpha)} .
$$

1.2. It is well known that $\mathfrak{g}^{\mathrm{c}}$ has the root space decomposition (see [7, p. 273])

$$
\mathfrak{g}^{\mathbf{c}}=\mathfrak{g}^{\mathbf{c}} \oplus \bigoplus_{\alpha \in \Phi} \mathfrak{g}_{\alpha}^{\mathbf{c}}
$$

where $\mathfrak{g}_{\alpha}^{\mathbf{c}}$ denotes the root subspace of $\mathfrak{g}^{\mathbf{c}}$ corresponding to $\alpha \in \Phi$.

Assuming $l \geq 2$, we choose $j_{0} \in I$, and then remove $\alpha_{j_{0}}$ from $\Delta$ to obtain

$$
\Delta_{0}=\left\{\alpha_{j}: j \in I_{0}\right\}, \quad \text { where } I_{0}=I \backslash\left\{j_{0}\right\} .
$$

Set $\Phi_{0}^{+}=\left\{\alpha \in \Phi^{+}: n_{j_{0}}(\alpha)=0\right\}$, and put $\Phi_{0}=\Phi_{0}^{+} \cup-\Phi_{0}^{+}$. Clearly $\Phi_{0}=-\Phi_{0}$ and $\sigma_{\alpha} \Phi_{0}=\Phi_{0}$ for all $\sigma_{\alpha}\left(\alpha \in \Delta_{0}\right)$. This shows that 
$\Phi_{0}$ is a root system (see [7, p. 370]). Let $\mathfrak{h}_{0}$ be the subspace of $\mathfrak{h}$ spanned by $H_{\alpha} \quad\left(\alpha \in \Phi_{0}\right)$. Then one may verify that

$$
\mathfrak{g}_{0}^{\mathbf{c}}=\mathfrak{h}_{0}^{\mathbf{c}} \oplus \bigoplus_{\alpha \in \Phi_{0}} \mathfrak{g}_{\alpha}^{\mathbf{c}}
$$

is a semisimple subalgebra of $\mathfrak{g}^{\mathbf{c}}$, with maximal toral subalgebra $\mathfrak{h}_{0}^{\mathbf{c}}$ (see [7, Ex. 30 of Ch. 4]). Evidently $\Phi_{0}$ is the root system of $\left(\mathfrak{g}_{0}^{\mathbf{c}}, \mathfrak{h}_{0}^{\mathbf{c}}\right)$, $\Delta_{0}$ is a base of $\Phi_{0}$, and $\Phi_{0}^{+}$is the set of positive roots with respect to $\Delta_{0}$.

Write $\Phi_{0}$ as a disjoint union of irreducible root systems, say

$$
\Phi_{0}=\Phi_{01} \cup \cdots \cup \Phi_{0 r} \text {. }
$$

Let $q \in\{1, \ldots, r\}$. Denote by $\mathfrak{h}_{0 q}$ the subspace of $\mathfrak{h}_{0}$ spanned by $H_{\alpha} \quad\left(\alpha \in \Phi_{0 q}\right)$. Then we find that

$$
\mathfrak{g}_{0 q}^{\mathbf{c}}=\mathfrak{h}_{0 q}^{\mathfrak{c}} \oplus \bigoplus_{\alpha \in \Phi_{0 q}} \mathfrak{g}_{\alpha}^{\mathbf{c}}
$$

is a simple ideal of $\mathfrak{g}_{0}^{\mathfrak{c}}$, with maximal toral subalgebra $\mathfrak{h}_{0 q}^{\mathfrak{c}}$. We also note that

and

$$
\mathfrak{h}_{0}^{\mathbf{c}}=\mathfrak{h}_{01}^{\mathbf{c}} \oplus \cdots \oplus \mathfrak{h}_{0 r}^{\mathbf{c}}
$$

$$
\mathfrak{g}_{0}^{\mathbf{c}}=\mathfrak{g}_{01}^{\mathbf{c}} \oplus \cdots \oplus \mathfrak{g}_{0 r}^{\mathbf{c}} .
$$

Now denote by $(\cdot, \cdot)_{0}$ and $(\cdot, \cdot)_{0 q}$ the inner products of $g_{0}$ and $g_{0 q}$ respectively. Then we have (see [3, Lemma 5.1])

$$
(\cdot, \cdot)_{0} \mid \mathfrak{g}_{0 q} \times \mathfrak{g}_{0 q}=(\cdot, \cdot)_{0 q},
$$

and so

$$
(X, Y)_{0}=\left(X_{1}, Y_{1}\right)_{01}+\cdots+\left(X_{r}, Y_{r}\right)_{0 r}
$$

for all $X=X_{1}+\cdots+X_{r}, Y=Y_{1}+\cdots+Y_{r} \in \mathfrak{g}_{0}$, with $X_{q}, Y_{q} \in \mathfrak{g}_{0 q}$. Further, since $\mathfrak{g}$ and $\mathfrak{g}_{0 q}$ are simple, there exists a positive constant $C_{q}$ satisfying (see [4, p. 242])

$$
(X, Y)_{0 q}=C_{q}(X, Y) \quad \forall X, Y \in \mathfrak{g}_{0 q} .
$$

We transfer these inner products to the corresponding dual spaces in the usual way.

Let $\Lambda_{0}^{+}$denote the set of dominant weights with respect to $\Delta_{0}$. We need to determine the set of fundamental dominant weights in $\Lambda_{0}^{\mp}$ : Suppose $\left\{\omega_{j}: j \in I\right\}$ is the set of fundamental dominant weights in $\Lambda^{+}$, for which (see $[3, \S 13.1]$ for definition)

$$
2 \frac{\left(\omega_{j}, \alpha_{k}\right)}{\left(\alpha_{k}, \alpha_{k}\right)}=\delta_{j k} \quad \forall j, k \in I .
$$


If we now set

$$
\tilde{\omega}_{j}=\omega_{j}-\operatorname{proj}_{\omega_{J_{0}}}\left(\omega_{j}\right) \quad \forall j \in I,
$$

then we have the following facts.

Fact 1. For each $j \in I_{0}, \tilde{\omega}_{j} \in \mathfrak{h}_{0 Q}^{*}$ whenever $\alpha_{j} \in \mathfrak{h}_{0 Q}^{*}$.

Proof. For all $j, k \in I_{0}$, we have

$$
\begin{aligned}
2 \frac{\left(\tilde{\omega}_{j}, \alpha_{k}\right)}{\left(\alpha_{k}, \alpha_{k}\right)} & =2 \frac{\left.\omega_{j}, \alpha_{k}\right)}{\left(\alpha_{k}, \alpha_{k}\right)}-2 \frac{\left(\operatorname{proj}_{\omega_{0}}\left(\omega_{j}\right), \alpha_{k}\right)}{\left(\alpha_{k}, \alpha_{k}\right)} \\
& =2 \frac{\left(\omega_{j}, \alpha_{k}\right)}{\left(\alpha_{k}, \alpha_{k}\right)}-2 \frac{\left(\omega_{j}, \omega_{j_{0}}\right)}{\left(\omega_{j_{0}}, \omega_{j_{0}}\right)} \frac{\left(\omega_{j_{0}}, \alpha_{k}\right)}{\left(\alpha_{k}, \alpha_{k}\right)} \\
& =2 \frac{\left(\omega_{j}, \alpha_{k}\right)}{\left(\alpha_{k}, \alpha_{k}\right)}-0=\delta_{j k} .
\end{aligned}
$$

Now take $j \in I_{0}$, and let $Q \in\{1, \ldots, r\}$ such that $\alpha_{j} \in \mathfrak{h}_{0 Q}^{*}$. Clearly

$$
\tilde{\omega}_{j} \perp \mathfrak{h}_{0 q}^{*} \quad \forall q \neq Q .
$$

Writing $\tilde{\omega}_{j}=\tilde{\omega}_{j 1}+\cdots+\tilde{\omega}_{j r}$, with $\tilde{\omega}_{j q} \in \mathfrak{h}_{0 q}^{*}$ for all $q \in\{1, \ldots, r\}$, we find that

$$
\tilde{\omega}_{j q}=0 \quad \forall q \neq Q
$$

We therefore have

$$
\tilde{\omega}_{j}=\tilde{\omega}_{j Q} \in \mathfrak{h}_{0 Q}^{*}
$$

as stated.

Fact 2. $\left\{\tilde{\omega}_{j}: j \in I_{0}\right\}$ is the set of fundamental dominant weights in $\Lambda_{0}^{+}$.

Proof. Take $j, k \in I_{0}$. Suppose $\tilde{\omega}_{j} \in \mathfrak{h}_{0 q}^{*}$ and $\alpha_{k} \in \mathfrak{h}_{0 q^{\prime}}^{*}$ for some $q, q^{\prime} \in\{1, \ldots, r\}$. If $q \neq q^{\prime}$, then clearly $\left(\tilde{\omega}_{j}, \alpha_{k}\right)_{0}=0$; otherwise we have

$$
2 \frac{\left(\tilde{\omega}_{j}, \alpha_{k}\right)_{0}}{\left(\alpha_{k}, \alpha_{k}\right)_{0}}=2 \frac{\left(\tilde{\omega}_{j}, \alpha_{k}\right)_{0 q}}{\left(\alpha_{k}, \alpha_{k}\right)_{0 q}}=2 \frac{\left(\tilde{\omega}_{j}, \alpha_{k}\right)}{\left(\alpha_{k}, \alpha_{k}\right)}=\delta_{j k} .
$$

Using Fact 1 , the assertion follows.

Fact 3. Suppose $\lambda=\sum_{j \in I} n_{j} \omega_{j} \in \Lambda^{+}$. Then $\lambda$ can be rewritten as

$$
\lambda=\lambda_{0}+\lambda_{1}
$$

where $\lambda_{0}=\sum_{j \in I_{0}} n_{j} \tilde{\omega}_{j} \in \Lambda_{0}^{+}$(with the same $n_{j}$ 's) and $\lambda_{1}=\operatorname{proj}_{\omega_{J_{0}}}(\lambda)$. 
Proof. Noting that $\tilde{\omega}_{j_{0}}=0$, we have

$$
\begin{aligned}
\lambda & =\sum_{j \in I} n_{j} \omega_{j}=\sum_{j \in I} n_{j} \tilde{\omega}_{j}+\sum_{j \in I} n_{j} \operatorname{proj}_{\omega_{j_{0}}}\left(\omega_{j}\right) \\
& =\sum_{j \in I_{0}} n_{j} \tilde{\omega}_{j}+\sum_{j \in I} n_{j} \frac{\left(\omega_{j}, \omega_{j_{0}}\right)}{\left(\omega_{j_{0}}, \omega_{j_{0}}\right)} \omega_{j_{0}} \\
& =\sum_{j \in I_{0}} n_{j} \tilde{\omega}_{j}+\frac{\left(\sum_{j \in I} n_{j} \omega_{j}, \omega_{j_{0}}\right)}{\left(\omega_{j_{0}}, \omega_{j_{0}}\right)} \omega_{j_{0}} \\
& =\sum_{j \in I_{0}} n_{j} \tilde{\omega}_{j}+\frac{\left(\lambda, \omega_{j_{0}}\right)}{\left(\omega_{j_{0}}, \omega_{j_{0}}\right)} \omega_{j_{0}} \\
& =\sum_{j \in I_{0}} n_{j} \tilde{\omega}_{j}+\operatorname{proj}_{\omega_{j_{0}}}(\lambda)=\lambda_{0}+\lambda_{1},
\end{aligned}
$$

as claimed.

REMARK. It is well known that the special element $\rho$ is a dominant weight in $\Lambda^{+}$. Indeed, $\rho=\sum_{j \in I} \omega_{j}$ (see [3, Lemma 13.3A]). By Fact 3 , we may rewrite $\rho=\rho_{0}+\rho_{1}$ where $\rho_{0}=\sum_{j \in I_{0}} \tilde{\omega}_{j} \in \Lambda_{0}^{+}$and $\rho_{1}=\operatorname{proj}_{\omega_{J_{0}}}(\rho)$. But then $\rho_{0}=\frac{1}{2} \sum_{\alpha \in \Phi_{0}^{+}} \alpha$, giving $\rho_{1}=\frac{1}{2} \sum_{\alpha \in \Phi_{1}^{+}} \alpha$ where $\Phi_{1}^{+}=\Phi^{+} \backslash \Phi_{0}^{+}$. As another consequence, we also have $\rho_{1}=$ $c \omega_{j_{0}}$ for some $c>0$. But we know that $2\left(\omega_{j_{0}}, \alpha_{j_{0}}\right) /\left(\alpha_{j_{0}}, \alpha_{j_{0}}\right)=1$, and so we find $c=2\left(\rho_{1}, \alpha_{j_{0}}\right) /\left(\alpha_{j_{0}}, \alpha_{j_{0}}\right)$. Hence we determine $\omega_{j_{0}}=$ $\frac{1}{2}\left(\left(\alpha_{j_{0}}, \alpha_{j_{0}}\right) /\left(\rho_{1}, \alpha_{j_{0}}\right)\right) \rho_{1}$, with $\rho_{1}=\frac{1}{2} \sum_{\alpha \in \Phi_{1}^{+}} \alpha$. This offers a method of finding the fundamental dominant weight $\omega_{j_{0}}$ for any given $j_{0} \in I$.

Introduce $\mathfrak{h}_{1}=\left\{H \in \mathfrak{h}: \alpha(H)=0 \quad \forall \alpha \in \Delta_{0}\right\}$. Obviously $\mathfrak{h}_{1}$ is a subalgebra of $\mathfrak{h}$, which is spanned by $H_{\rho_{1}}$ (by the above remark). Moreover, we have (like Fact 3)

Fact 4. Every $H \in \mathfrak{h}$ can be written as

$$
H=H_{0}+H_{1}
$$

where $H_{0} \in \mathfrak{h}_{0}$ and $H_{1} \in \mathfrak{h}_{1}$.

REMARK. $H_{0} \in \mathfrak{h}_{0}$ means that $H_{0}=H_{\nu_{0}}$, where $\nu_{0} \in \operatorname{span}\left(\Delta_{0}\right)$, while $H_{1} \in \mathfrak{h}_{1}$ means that $H_{1}=H_{\nu_{1}}$, where $\nu_{1}=r \rho_{1}$ for some $r \in \mathbf{R}$. Thus clearly $\mathfrak{h}_{0} \perp \mathfrak{h}_{1}$, and so Fact 4 actually states that $\mathfrak{h}=\mathfrak{h}_{0} \oplus \mathfrak{h}_{1}$.

Suppose we are in $\left(\mathfrak{g}_{0}, \mathfrak{h}_{0}\right)$. To each $\lambda_{0} \in \Lambda_{0}^{+}$, we associate the representation $\tilde{\pi}_{\lambda_{0}}$, the set of weights $\tilde{\varpi}_{\lambda_{0}}$, the character $\tilde{\chi}_{\lambda_{0}}$, and the 
dimension $\tilde{d}_{\lambda_{0}}$. For all $\lambda_{0} \in \Lambda_{0}^{+}$and $H_{0} \in \mathfrak{h}_{0}$, we have

$$
\tilde{\chi}_{\lambda_{0}}\left(\exp \left(H_{0}\right)\right)=\sum_{\lambda^{\prime} \in \tilde{\omega}_{\lambda_{0}}} \tilde{m}_{\lambda_{0}}\left(\lambda^{\prime}\right) \exp \left(i \lambda^{\prime}\left(H_{0}\right)\right)
$$

and

$$
\tilde{d}_{\lambda_{0}}=\sum_{\lambda^{\prime} \in \tilde{\varpi}_{\lambda_{0}}} \tilde{m}_{\lambda_{0}}\left(\lambda^{\prime}\right),
$$

with $\tilde{m}_{\lambda_{0}}\left(\lambda^{\prime}\right) \in \mathbf{Z}^{+}$being the multiplicity of $\lambda^{\prime}$ in $\tilde{\pi}_{\lambda_{0}}$.

Let $\mathscr{W}_{0}$ (or $\mathscr{W}\left[\Delta_{0}\right]$ if necessary) denote the subgroup of $\mathscr{W}$ generated by $\sigma_{\alpha}\left(\alpha \in \Delta_{0}\right)$. The Weyl formulae then read

$$
\tilde{\chi}_{\lambda_{0}}\left(\exp \left(H_{0}\right)\right)=\frac{\sum_{r \in \mathscr{W}_{0}} \operatorname{det}(\tau) \exp \left(i \tau\left(\lambda_{0}+\rho_{0}\right)\left(H_{0}\right)\right)}{\prod_{\alpha \in \Phi_{0}^{+}} 2 i \sin \frac{1}{2} \alpha\left(H_{0}\right)}
$$

and

$$
\tilde{d}_{\lambda_{0}}=\prod_{\alpha \in \Phi_{0}^{+}} \frac{\left(\lambda_{0}+\rho_{0}, \alpha\right)}{\left(\rho_{0}, \alpha\right)} .
$$

We should note that the inner product in the expression above is really the inner product of $\mathfrak{g}$. Indeed, we may calculate

$$
\begin{aligned}
\tilde{d}_{\lambda_{0}} & =\lim _{s \rightarrow 0} \tilde{\chi}_{\lambda_{0}}\left(\exp \left(s H_{\rho_{0}}\right)\right) \\
& =\lim _{s \rightarrow 0} \frac{\sum_{\tau \in \mathscr{W}_{0}} \operatorname{det}(\tau) \exp \left(i \tau\left(\lambda_{0}+\rho_{0}\right)\left(s H_{\rho_{0}}\right)\right)}{\prod_{\alpha \in \Phi_{0}^{+}} 2 i \sin \frac{1}{2} \alpha\left(s H_{\rho_{0}}\right)} \\
& =\lim _{s \rightarrow 0} \frac{\sum_{\tau \in \mathscr{W}_{0}} \operatorname{det}(\tau) \exp \left(i \tau \rho_{0}\left(s H_{\lambda_{0}+\rho_{0}}\right)\right)}{\prod_{\alpha \in \Phi_{0}^{+}} 2 i \sin \frac{1}{2} \alpha\left(s H_{\rho_{0}}\right)} \\
& =\lim _{s \rightarrow 0} \frac{\prod_{\alpha \in \Phi_{0}^{+}} 2 i \sin \frac{1}{2} \alpha\left(s H_{\lambda_{0}+\rho_{0}}\right)}{\prod_{\alpha \in \Phi_{0}^{+}} 2 i \sin \frac{1}{2} \alpha\left(s H_{\rho_{0}}\right)} \\
& =\prod_{\alpha \in \Phi_{0}^{+}} \frac{\alpha\left(H_{\lambda_{0}+\rho_{0}}\right)}{\alpha\left(H_{\rho_{0}}\right)}=\prod_{\alpha \in \Phi_{0}^{+}} \frac{\left(\lambda_{0}+\rho_{0}, \alpha\right)}{\left(\rho_{0}, \alpha\right)}
\end{aligned}
$$

(see [8, p. 106] for clarification).

Allowing $\mathscr{W}$ to act, one may observe that all the above facts still hold for the system constituted by $\sigma \Phi_{0}(\sigma \in \mathscr{W})$, as well as for that by $\Phi_{0}$. Moreover, the two facts below explain the connection between one system and another.

Fact 5. $\sigma \mathscr{W}\left[\Delta_{0}\right] \sigma^{-1}=\mathscr{W}\left[\sigma \Delta_{0}\right]$ for any $\sigma \in \mathscr{W}$. 
Proof. Obvious (see [3, Lemma 9.2] for justification).

Fact 6. $\tilde{\chi}_{\sigma \lambda_{0}}\left(\exp \left(H_{\sigma \nu_{0}}\right)\right)=\tilde{\chi}_{\lambda_{0}}\left(\exp \left(H_{\nu_{0}}\right)\right)$ for any $\sigma \in \mathscr{W}$.

Proof. For any $\sigma \in \mathscr{W}$, we have (by Fact 5)

$$
\begin{aligned}
\tilde{\chi}_{\sigma \lambda_{0}}\left(\exp \left(H_{\sigma \nu_{0}}\right)\right) & =\frac{\sum_{\tau \in \mathscr{W}\left[\sigma \Delta_{0}\right]} \operatorname{det}(\tau) \exp \left(i \tau\left(\sigma \lambda_{0}+\sigma \rho_{0}\right)\left(H_{\sigma \nu_{0}}\right)\right)}{\prod_{\alpha \in \sigma \Phi_{0}^{+}} 2 i \sin \frac{1}{2} \alpha\left(H_{\sigma \nu_{0}}\right)} \\
& =\frac{\sum_{\tau \in \sigma \mathscr{W}\left[\Delta_{0}\right] \sigma^{-1}} \operatorname{det}(\tau) \exp \left(i \tau \sigma\left(\lambda_{0}+\rho_{0}\right)\left(H_{\sigma \nu_{0}}\right)\right)}{\prod_{\alpha \in \sigma \Phi_{0}^{+}} 2 i \sin \frac{1}{2} \alpha\left(H_{\sigma \nu_{0}}\right)} \\
& =\frac{\sum_{\tau \in \mathscr{W}\left[\Delta_{0}\right]} \operatorname{det}\left(\sigma \tau \sigma^{-1}\right) \exp \left(i \sigma \tau\left(\lambda_{0}+\rho_{0}\right)\left(H_{\sigma \nu_{0}}\right)\right)}{\prod_{\alpha \in \Phi_{0}^{+}} 2 i \sin \frac{1}{2} \sigma \alpha\left(H_{\sigma \nu_{0}}\right)} \\
& =\frac{\sum_{\tau \in \mathscr{W}\left[\Delta_{0}\right]} \operatorname{det}(\tau) \exp \left(i \tau\left(\lambda_{0}+\rho_{0}\right)\left(H_{\nu_{0}}\right)\right)}{\prod_{\alpha \in \Phi_{0}^{+}} 2 i \sin \frac{1}{2} \alpha\left(H_{\nu_{0}}\right)} \\
& =\tilde{\chi} \lambda_{0}\left(\exp \left(H_{\nu_{0}}\right)\right),
\end{aligned}
$$

as stated.

2. The proof of the theorem. The outline of the proof is as follows. We first look for an estimate for all $s \in(0, R)$, then examine the decay for large $s$, and finally combine the results. The result obtained is valid under the assumption that $G$ is simple, but then it extends to every semisimple Lie group $G$.

2.1. For all $s \in(0, R), \lambda \in \Lambda^{+}$, we have (see [2, p. 813])

$$
\hat{\mu}_{s H}(\lambda)=\frac{\chi_{\lambda}(\exp (s H))}{d \lambda} .
$$

Using the multiplicity formulae, we write

$$
\hat{\mu}_{s H}(\lambda)=\frac{\sum_{\lambda^{\prime} \in \varpi_{\lambda}} m_{\lambda}\left(\lambda^{\prime}\right) \exp \left(i \lambda^{\prime}(s H)\right)}{\sum_{\lambda^{\prime} \in \varpi_{\lambda}} m_{\lambda}\left(\lambda^{\prime}\right)} .
$$

Hence, we have

$$
\begin{aligned}
\left|\left(\frac{\partial}{\partial s}\right)^{k} \hat{\mu}_{s H}(\lambda)\right| & \leq \frac{\sum_{\lambda^{\prime} \in \varpi_{\lambda}} m_{\lambda}\left(\lambda^{\prime}\right)\left|\left(\frac{\partial}{\partial s}\right)^{k} \exp \left(i \lambda^{\prime}(s H)\right)\right|}{\sum_{\lambda^{\prime} \in \varpi_{\lambda}} m_{\lambda}\left(\lambda^{\prime}\right)} \\
& \leq|H|^{k}|\lambda|^{k}=C_{k}(H)|\lambda|^{k}
\end{aligned}
$$

for all $k=0,1,2, \ldots$. 
2.2. By the Weyl formulae, for all $s \in(0, R), \lambda \in \Lambda^{+}$, we have

$$
\hat{\mu}_{s H}(\lambda)=\frac{\sum_{\sigma \in \mathscr{W}} \operatorname{det}(\sigma) \exp (i(\lambda+\rho)(s H))}{\prod_{\alpha \in \Phi^{+}} 2 i \sin \frac{1}{2} \alpha(s H)} \prod_{\alpha \in \Phi^{+}} \frac{(\rho, \alpha)}{(\lambda+\rho, \alpha)} .
$$

In the case $l=1$, one can easily obtain

$$
\left|\left(\frac{\partial}{\partial s}\right)^{k} \hat{\mu}_{s H}(\lambda)\right| \leq C_{k}(H) \frac{|\lambda+\rho|^{k}}{s|\lambda+\rho|},
$$

for all $k=0,1,2, \ldots$. So assume, hereafter, that $l \geq 2$.

For each $\lambda \in \Lambda^{+}$, choose $j_{0} \in I$ for which $\left(\lambda+\rho, \alpha_{j_{0}}\right)$ is maximal. As before, we write $\Delta_{0}=\Delta \backslash\left\{\alpha_{j_{0}}\right\}, \Phi_{0}^{+}=\left\{\alpha \in \Phi^{+}: n_{j_{0}}(\alpha)=0\right\}$, and $\Phi_{1}^{+}=\left\{\alpha \in \Phi^{+}: n_{j_{0}}(\alpha) \geq 1\right\}$. (Note that $\Phi_{1}^{+}=\Phi^{+} \backslash \Phi_{0}^{+}$, and that $\Phi_{1}^{+}$depends on the choice of $j_{0}$, and so depends on $\lambda$.) Clearly, if $\alpha \in \Phi_{0}^{+}$, then

$$
(\lambda+\rho, \alpha) \geq(\rho, \alpha) \geq C,
$$

and if $\alpha \in \Phi_{1}^{+}$, then (by the choice of $j_{0}$ )

$$
(\lambda+\rho, \alpha) \geq n_{j_{0}}(\alpha)\left(\lambda+\rho, \alpha_{j_{0}}\right) \geq C|\lambda+\rho| .
$$

Moreover,

$$
\gamma=\min _{j \in I}\left|\left\{\alpha \in \Phi^{+}: n_{j}(\alpha) \geq 1\right\}\right| \leq\left|\Phi_{1}^{+}\right| .
$$

Recall that $\mathscr{W}_{0}$ is the subgroup of $\mathscr{W}$ generated by $\sigma_{\alpha}\left(\alpha \in \Delta_{0}\right)$. For an appropriate $\mathscr{S} \subset \mathscr{W}$, we write $\mathscr{W}=\bigcup_{\sigma \in \mathscr{S}} \sigma \mathscr{W}_{0}$ (disjoint union). We then obtain

$\hat{\mu}_{s H}(\lambda)=\sum_{\sigma \in \mathscr{S}}\left(\frac{\sum_{\tau \in \mathscr{W}_{0}} \operatorname{det}(\sigma \tau) \exp (i \sigma \tau(\lambda+\rho)(s H))}{\prod_{\alpha \in \Phi^{+}} 2 i \sin \frac{1}{2} \alpha(s H)} \prod_{\alpha \in \Phi^{+}} \frac{(\rho, \alpha)}{(\lambda+\rho, \alpha)}\right)$.

For each reflection $\sigma_{\alpha} \in \mathscr{W}$, we know that $\operatorname{det}\left(\sigma_{\alpha}\right)=-1, \sigma_{\alpha} \alpha=-\alpha$, and $\sigma_{\alpha}\left(\Phi^{+} \backslash\{\alpha\}\right)=\Phi^{+} \backslash\{\alpha\}$ (see [3, Lemma 10.2B]). Thus, for any $\sigma \in \mathscr{W}$, we have

$$
\prod_{\alpha \in \Phi^{+}} 2 i \sin \frac{1}{2} \alpha(s H)=\operatorname{det}(\sigma) \prod_{\alpha \in \Phi^{+}} 2 i \sin \frac{1}{2} \sigma \alpha(s H) .
$$

It follows that

$$
\begin{aligned}
\hat{\mu}_{s H}(\lambda)= & \sum_{\sigma \in \mathscr{S}}\left(\frac{\sum_{\tau \in \mathscr{W}_{0}} \operatorname{det}(\tau) \exp (i \sigma \tau(\lambda+\rho)(s H))}{\prod_{\alpha \in \Phi_{0}^{+}} 2 i \sin \frac{1}{2} \sigma \alpha(s H)} \prod_{\alpha \in \Phi_{0}^{+}} \frac{(\rho, \alpha)}{(\lambda+\rho, \alpha)}\right) \\
& \times\left(\prod_{\alpha \in \Phi_{1}^{+}} \frac{1}{2 i \sin \frac{1}{2} \sigma \alpha(s H)} \frac{(\rho, \alpha)}{(\lambda+\rho, \alpha)}\right) .
\end{aligned}
$$


Now fix $\sigma \in \mathscr{S}$. We write $H=H_{\sigma \nu}$, with $\nu=\nu_{0}+\nu_{1}$, where $\nu_{0} \in \operatorname{span}\left(\Delta_{0}\right)$ and $\nu_{1}=r \rho_{1}$ for some $r \in \mathbf{R}$. Then put $H_{0}=H_{\nu_{0}}$ and $H_{1}=H_{\nu_{1}}$. Next recall that $\Lambda_{0}^{+}$is the set of dominant weights corresponding to $\Phi_{0}^{+}$. For each $\lambda \in \Lambda^{+}$, we write $\lambda=\lambda_{0}+\lambda_{1}$, where $\lambda_{0} \in \Lambda_{0}^{+}$and $\lambda_{1}=c \rho_{1}$ for some $c \in \mathbf{R}^{+}$. Hence, for all $\alpha \in \Phi_{0}^{+}$, we have $(\rho, \alpha)=\left(\rho_{0}, \alpha\right)$ and $(\lambda+\rho, \alpha)=\left(\lambda_{0}+\rho_{0}, \alpha\right)$. Further, for all $\alpha \in \Phi_{0}^{+}$,

$$
\begin{aligned}
\sigma \alpha(H) & =(\sigma \alpha, \sigma \nu)=(\alpha, \nu) \\
& =\left(\alpha, \nu_{0}+\nu_{1}\right)=\left(\alpha, \nu_{0}\right) \quad\left(\text { as } \nu_{1} \perp \alpha\right) \\
& =\alpha\left(H_{\nu_{0}}\right)=\alpha\left(H_{0}\right),
\end{aligned}
$$

and whenever $\tau \in \mathscr{W}_{0}$,

$$
\begin{aligned}
\sigma \tau(\lambda+\rho)(H) & =(\sigma \tau(\lambda+\rho), \sigma \nu)=(\tau(\lambda+\rho), \nu) \\
& =\left(\tau\left(\lambda_{0}+\rho_{0}\right)+\tau\left(\lambda_{1}+\rho_{1}\right), \nu_{0}+\nu_{1}\right) \\
& =\left(\tau\left(\lambda_{0}+\rho_{0}\right)+\left(\lambda_{1}+\rho_{1}\right), \nu_{0}+\nu_{1}\right) \quad\left(\text { as } \tau \in \mathscr{W}_{0}\right) \\
& =\left(\tau\left(\lambda_{0}+\rho_{0}\right), \nu_{0}\right)+\left(\lambda_{1}+\rho_{1}, \nu_{1}\right) \quad \text { (by orthogonality) } \\
& =\tau\left(\lambda_{0}+\rho_{0}\right)\left(H_{\nu_{0}}\right)+\left(\lambda_{1}+\rho_{1}\right)\left(H_{\nu_{1}}\right) \\
& =\tau\left(\lambda_{0}+\rho_{0}\right)\left(H_{0}\right)+\left(\lambda_{1}+\rho_{1}\right)\left(H_{1}\right) .
\end{aligned}
$$

It turns out that

$$
\begin{aligned}
\frac{\sum_{\tau \in \mathscr{W}_{0}} \operatorname{det}(\tau) \exp (i \sigma \tau(\lambda+\rho)(s H))}{\prod_{\alpha \in \Phi_{0}^{+}} 2 i \sin \frac{1}{2} \sigma \alpha(s H)} \prod_{\alpha \in \Phi_{0}^{+}} \frac{(\rho, \alpha)}{(\lambda+\rho, \alpha)} \\
=\exp \left(i\left(\lambda_{1}+\rho_{1}\right)\left(s H_{1}\right)\right) \frac{\sum_{\tau \in \mathscr{W}_{0}} \operatorname{det}(\tau) \exp \left(i \tau\left(\lambda_{0}+\rho_{0}\right)\left(s H_{0}\right)\right)}{\prod_{\alpha \in \Phi_{0}^{+}} 2 i \sin \frac{1}{2} \alpha\left(s H_{0}\right)} \\
\quad \times \prod_{\alpha \in \Phi_{0}^{+}} \frac{\left(\rho_{0}, \alpha\right)}{\left(\lambda_{0}+\rho_{0}, \alpha\right)} \\
=\exp \left(i\left(\lambda_{1}+\rho_{1}\right)\left(s H_{1}\right)\right) \frac{\tilde{\chi}_{\lambda_{0}}\left(\exp \left(s H_{0}\right)\right)}{\tilde{d}_{\lambda_{0}}} \\
=\exp \left(i\left(\lambda_{1}+\rho_{1}\right)\left(s H_{1}\right)\right) \frac{\sum_{\lambda^{\prime} \in \tilde{\varpi}_{\lambda_{0}}} \tilde{m}_{\lambda_{0}}\left(\lambda^{\prime}\right) \exp \left(i \lambda^{\prime}\left(s H_{0}\right)\right)}{\sum_{\lambda^{\prime} \in \tilde{\varpi}_{\lambda_{0}}} \tilde{m}_{\lambda_{0}}\left(\lambda^{\prime}\right)} \\
=\frac{\sum_{\lambda^{\prime} \in \tilde{\varpi}_{\lambda_{0}}} \tilde{m}_{\lambda_{0}}\left(\lambda^{\prime}\right) \exp \left(i\left(\lambda^{\prime}+\lambda_{1}+\rho_{1}\right)(s H)\right)}{\sum_{\lambda^{\prime} \in \tilde{\varpi}_{\lambda_{0}}} \tilde{m}_{\lambda_{0}}\left(\lambda^{\prime}\right)} \text { (by orthogonality). }
\end{aligned}
$$

So we have 


$$
\begin{aligned}
\hat{\mu}_{s H}(\lambda)= & \sum_{\sigma \in \mathscr{S}}\left(\frac{\sum_{\lambda^{\prime} \in \tilde{\varpi}_{\lambda_{0}}} \tilde{m}_{\lambda_{0}}\left(\lambda^{\prime}\right) \exp \left(i\left(\lambda^{\prime}+\lambda_{1}+\rho_{1}\right)(s H)\right)}{\sum_{\lambda^{\prime} \in \tilde{\varpi}_{\lambda_{0}}} \tilde{m}_{\lambda_{0}}\left(\lambda^{\prime}\right)}\right) \\
& \times\left(\prod_{\alpha \in \Phi_{1}^{+}} \frac{1}{\sigma \alpha(s H)} \frac{\sigma \alpha(s H)}{2 i \sin \frac{1}{2} \sigma \alpha(s H)} \frac{(\rho, \alpha)}{(\lambda+\rho, \alpha)}\right) .
\end{aligned}
$$

For all $k=0,1,2, \ldots$, we have the estimates

$$
\left|\left(\frac{\partial}{\partial s}\right)^{k} \frac{\sum_{\lambda^{\prime} \in \tilde{\varpi}_{\lambda_{0}}} \tilde{m}_{\lambda_{0}}\left(\lambda^{\prime}\right) \exp \left(i\left(\lambda^{\prime}+\lambda_{1}+\rho_{1}\right)(s H)\right)}{\sum_{\lambda^{\prime} \in \tilde{\varpi}_{\lambda_{0}}} \tilde{m}_{\lambda_{0}}\left(\lambda^{\prime}\right)}\right| \leq|H|^{k}|\lambda+\rho|^{k}
$$

$$
\begin{gathered}
\left|\left(\frac{\partial}{\partial s}\right)^{k} \prod_{\alpha \in \Phi_{1}^{+}} \sigma \alpha(s H)^{-1}\right| \leq C_{k}(H) s^{-k-\left|\Phi_{1}^{+}\right|}, \\
\left|\left(\frac{\partial}{\partial s}\right)^{k} \prod_{\alpha \in \Phi_{1}^{+}} \frac{\sigma \alpha(s H)}{2 i \sin \frac{1}{2} \sigma \alpha(s H)}\right| \leq C_{k} \quad \text { (by Leibniz' rule), } \\
\left|\prod_{\alpha \in \Phi_{1}^{+}} \frac{(\rho, \alpha)}{(\lambda+\rho, \alpha)}\right| \leq C|\lambda+\rho|^{-\left|\Phi_{1}^{+}\right|} \\
\quad\left(\text { as }(\lambda+\rho, \alpha) \geq C|\lambda+\rho| \text { for all } \alpha \in \Phi_{1}^{+}\right) .
\end{gathered}
$$

Therefore, by Leibniz' rule for the derivatives of products, we obtain

$$
\begin{aligned}
& \left|\left(\frac{\partial}{\partial s}\right)^{k} \hat{\mu}_{s H}(\lambda)\right| \\
& \leq \sum_{\sigma \in \mathscr{S}} \sum_{k_{1}+k_{2}+k_{3}=k} C_{k_{1}, k_{2}, k_{3}} \mid\left(\frac{\partial}{\partial s}\right)^{k_{1}}(1 \text { st term }) \mid \\
& \times \mid\left(\frac{\partial}{\partial s}\right)^{k_{2}}(2 \text { nd term })||\left(\frac{\partial}{\partial s}\right)^{k_{3}}(3 \text { rd term }) \mid \text { |4th term } \mid \\
& \leq \sum_{\sigma \in \mathscr{S}} \sum_{k_{1}+k_{2}+k_{3}=k} C_{k_{1}, k_{2}, k_{3}}(H)|H|^{k_{1}}|\lambda+\rho|^{k_{1}} S^{-k_{2}}(s|\lambda+\rho|)^{-\left|\Phi_{1}^{+}\right|} \\
& \leq C_{k}(H)(1+|H|)^{k} \frac{|\lambda+\rho|^{k}}{(s|\lambda+\rho|)^{\left|\Phi_{1}^{+}\right|}} \quad(\text { provided } s|\lambda+\rho|>1) \\
& \leq C_{k}(H) \frac{|\lambda+\rho|^{k}}{(s|\lambda+\rho|)^{\gamma}} \quad\left(\text { as } \gamma \leq\left|\Phi_{1}^{+}\right|\right),
\end{aligned}
$$

for all $k=0,1,2, \ldots$, as desired. 
Combining this with the previous estimate, we obtain the result.

2.3. We shall now extend our result to every semisimple Lie group $G$. The key is to prove that Fact 2 in $\S 1.2$ is still valid.

Let us write $\Phi$ as a disjoint union of irreducible root systems

$$
\Phi=\Phi^{(1)} \cup \cdots \cup \Phi^{(n)},
$$

and split $\Delta$ into

$$
\Delta=\Delta^{(1)} \cup \cdots \cup \Delta^{(n)},
$$

with $\Delta^{(m)}=\Delta \cap \Phi^{(m)}$ being a base of $\Phi^{(m)}$ for each $m \in\{1, \ldots, n\}$. The Lie algebra $\mathfrak{g}^{\mathbf{c}}$ is now a direct sum of simple ideals

$$
\mathfrak{g}^{\mathbf{c}}=\mathfrak{g}^{(1) \mathbf{c}} \oplus \cdots \oplus \mathfrak{g}^{(n) \mathbf{c}} .
$$

As before, we choose $j_{0} \in I$ and remove $\alpha_{j_{0}}$ from $\Delta$ to obtain

$$
\Delta_{0}=\Delta \backslash\left\{\alpha_{j_{0}}\right\} \text {. }
$$

But $\alpha_{j_{0}} \in \Delta^{(M)}$ for some $M \in\{1, \ldots, n\}$, and so

$$
\Delta_{0}=\Delta^{(1)} \cup \cdots \cup \Delta_{0}^{(M)} \cup \cdots \cup \Delta^{(n)},
$$

with $\Delta_{0}^{(M)}=\Delta^{(M)} \backslash\left\{\alpha_{j_{0}}\right\}$. The Lie algebra $\mathfrak{g}_{0}$ (as in $\left.\S 1.2\right)$ then decomposes into

$$
\mathfrak{g}_{0}^{\mathbf{c}}=\mathfrak{g}^{(1) \mathbf{c}} \oplus \cdots \oplus \mathfrak{g}_{0}^{(M) \mathbf{c}} \oplus \cdots \oplus \mathfrak{g}^{(n) \mathbf{c}},
$$

where $\mathfrak{g}_{0}^{(M) \mathbf{c}}$ is the Lie subalgebra corresponding to $\Delta_{0}^{(M)}$. Now let $K, K_{0}, K^{(m)}$, and $K_{0}^{(M)}$ denote the Killing forms of $\mathfrak{g}, \mathfrak{g}_{0}, \mathfrak{g}^{(m)}$, and $\mathfrak{g}_{0}^{(M)}$ respectively. Then, for each $m \in\{1, \ldots, n\}, m \neq M$, we have

$$
\left.K_{0}\right|_{\mathfrak{g}^{(m)} \times \mathfrak{g}^{(m)}}=K^{(m)}=\left.K\right|_{\mathfrak{g}^{(m)} \times \mathfrak{g}^{(m)}} ;
$$

while for $m=M$, the connection between $K^{(M)}$ and $K_{0}^{(M)}$ is explained in $\S 1.2$. We therefore find that Fact 2 still holds, and thus the extension is clear.

3. An example: The sharpness of the estimate. We shall here consider an example concerning the sharpness of the $L^{p}$-estimate.

Let $G=\mathbf{S U}(2)$, the Lie group consisting of $2 \times 2$ complex matrices of the form

$$
\left(\begin{array}{cc}
\alpha & \beta \\
-\bar{\beta} & \bar{\alpha}
\end{array}\right)
$$

with $|\alpha|^{2}+|\beta|^{2}=1$. Its Lie algebra $\mathfrak{g}$ then contains all matrices of the form

$$
\left(\begin{array}{cc}
i a & b \\
-\bar{b} & -i a
\end{array}\right)
$$


with $a \in \mathbf{R}, b \in \mathbf{C}$. Here $\gamma=1$ and the special element is

$$
H_{\rho}=\left(\begin{array}{cc}
i & 0 \\
0 & -i
\end{array}\right) \text {. }
$$

In $\mathfrak{g}$, one may define the norm $|\cdot|$ by

$$
\left|\left(\begin{array}{cc}
i a & b \\
-\bar{b} & -i a
\end{array}\right)\right|=\left(a^{2}+|b|^{2}\right)^{1 / 2} \quad \forall a \in \mathbf{R}, b \in \mathbf{C} .
$$

For any $y \in G, X \in \mathfrak{g}$, one may observe that

$$
X^{\prime}=y X y^{-1} \in \mathfrak{g},
$$

with $\left|X^{\prime}\right|=|X|$. Conversely, for any $X, X^{\prime} \in \mathfrak{g}$ with $|X|=\left|X^{\prime}\right|$, one can find $y \in G$ such that $X^{\prime}=y X y^{-1}$.

Denote by $B_{0}(\pi)$ the ball in $\mathfrak{g}$ which has centre 0 and radius $\pi$. It is then evident that the map exp: $B_{0}(\pi) \rightarrow G$ is injective. Indeed, for each $x \in G$, there exists a unique $X \in B_{0}(\pi)$ for which $x=\exp (X)$. Diagonalizing such an $X$, one has

$$
x=y \exp \left(\omega H_{\rho}\right) y^{-1}, \quad \text { where } \omega=|X|,
$$

for some $y \in G$. It is seen here that $\operatorname{trace}(x)=2 \cos \omega$.

As suggested in [6], let us consider the function $f: G \rightarrow \mathbf{R}^{+}$given by

$$
f(\exp (X))= \begin{cases}\frac{|X|^{-2}}{\log |X|^{-1}}, & \text { if } 0<|X|<\frac{1}{2}, \\ 0, & \text { otherwise. }\end{cases}
$$

One may observe that $f \in L^{p}(G)$, whenever $1 \leq p \leq \frac{3}{2}$. On the other hand, regarding the maximal function $\mathscr{M} f=\mathscr{M}_{H_{\rho}} f$, we claim that $\mathscr{M} f(x)=\infty$ for all $x \in G$.

Before verifying our claim, we remark that

$$
f(-\exp (X))=f\left(\exp \left(X^{\prime}\right)\right)
$$

where $\left|X^{\prime}\right|=\pi-|X|$. Moreover, $f\left(y x y^{-1}\right)=f(x)$ for all $x, y \in G$. In fact, for all $x, y \in G$, we have

$$
\begin{aligned}
f\left(y x y^{-1}\right) & =f\left(y \exp (X) y^{-1}\right) \quad(\text { for some } X \in \mathfrak{g}) \\
& =f\left(\exp \left(y X y^{-1}\right)\right) \\
& =f\left(\exp \left(X^{\prime}\right)\right) \quad\left(\text { where }\left|X^{\prime}\right|=|X|\right) \\
& =f(\exp (X))=f(x) .
\end{aligned}
$$


Similarly, we observe that $\mathscr{M} f\left(y x y^{-1}\right)=\mathscr{M} f(x)$ for all $x, y \in G$. To be precise, for all $x, y \in G$, we have

$$
\begin{aligned}
\mathscr{M} f\left(y x y^{-1}\right) & =\sup _{s \in(0, \pi)} \int_{G} f\left(y \times y^{-1} g \exp \left(s H_{\rho}\right) g^{-1}\right) d g \\
& =\sup _{s \in(0, \pi)} \int_{G} f\left(x y^{-1} g \exp \left(s H_{\rho}\right) g^{-1} y\right) d g \\
& =\sup _{s \in(0, \pi)} \int_{G} f\left(x g^{\prime} \exp \left(s H_{\rho}\right) g^{\prime-1}\right) d g^{\prime}=\mathscr{M} f(x) .
\end{aligned}
$$

We shall now verify our claim. First, for $x= \pm \mathbf{1}$, we have

$$
\begin{aligned}
\mathscr{M} f( \pm \mathbf{1}) & =\sup _{s \in(0, \pi)} \int_{G} f\left( \pm g \exp \left(s H_{\rho}\right) g^{-1}\right) d g \\
& =\sup _{s \in(0, \pi)} \int_{G} f\left( \pm \exp \left(s H_{\rho}\right)\right) d g=\sup _{s \in(0, \pi)} f\left( \pm \exp \left(s H_{\rho}\right)\right) \\
& =\sup _{s \in\left(0, \frac{1}{2}\right)} \frac{s^{-2}}{\log s^{-1}}=\infty .
\end{aligned}
$$

Next, for $x \neq \pm 1$, we may assume that $x=\exp \left(\frac{t}{2} H_{\rho}\right)$ for some $0<t<2 \pi$, and hence

$$
\begin{aligned}
\mathscr{M} f(x) & =\mathscr{M} f\left(\exp \left(\frac{t}{2} H_{\rho}\right)\right) \\
& =\sup _{s \in(0, \pi)} \int_{G} f\left(\exp \left(\frac{t}{2} H_{\rho}\right) g \exp \left(s H_{\rho}\right) g^{-1}\right) d g \\
& \geq \int_{G} f\left(\exp \left(\frac{t}{2} H_{\rho}\right) g \exp \left(\frac{t}{2} H_{\rho}\right) g^{-1}\right) d g .
\end{aligned}
$$

Writing each $g \in G$ as $g=h_{\theta} k_{\phi} h_{\theta^{\prime}}$, where $h_{\theta}=\exp \left(\frac{\theta}{2} H_{\rho}\right)$ and $k_{\phi}$ is the matrix of rotation with angle $\frac{\phi}{2}$, we have (see [9, pp. 99-100]) $\mathscr{M} f(x) \geq \frac{1}{16 \pi^{2}} \int_{-2 \pi}^{2 \pi} \int_{0}^{2 \pi} \int_{0}^{\pi} f\left(h_{t} h_{\theta} k_{\phi} h_{\theta^{\prime}} h_{t} h_{-\theta^{\prime}} k_{-\phi} h_{-\theta}\right) \sin \phi d \phi d \theta d \theta^{\prime}$

$$
\begin{aligned}
& =\frac{1}{16 \pi^{2}} \int_{-2 \pi}^{2 \pi} \int_{0}^{2 \pi} \int_{0}^{\pi} f\left(h_{t} h_{\theta} k_{\phi} h_{t} k_{-\phi} h_{-\theta}\right) \sin \phi d \phi d \theta d \theta^{\prime} \\
& =\frac{1}{4 \pi} \int_{0}^{2 \pi} \int_{0}^{\pi} f\left(h_{t} h_{\theta} k_{\phi} h_{t} k_{-\phi} h_{-\theta}\right) \sin \phi d \phi d \theta \\
& =\frac{1}{4 \pi} \int_{0}^{2 \pi} \int_{0}^{\pi} f\left(h_{-\theta} h_{t} h_{\theta} k_{\phi} h_{t} k_{-\phi}\right) \sin \phi d \phi d \theta \\
& =\frac{1}{4 \pi} \int_{0}^{2 \pi} \int_{0}^{\pi} f\left(h_{t} k_{\phi} h_{t} k_{-\phi}\right) \sin \phi d \phi d \theta \\
& =\frac{1}{2} \int_{0}^{\pi} f\left(h_{t} k_{\phi} h_{t} k_{-\phi}\right) \sin \phi d \phi .
\end{aligned}
$$


Let us now investigate the integrand. Multiplying out, we get

$$
h_{t} k_{\phi} h_{t} k_{-\phi}=\left(\begin{array}{cc}
e^{i t} \cos ^{2} \frac{\phi}{2}+\sin ^{2} \frac{\phi}{2} & \cos \frac{\phi}{2} \sin \frac{\phi}{2}\left(1-e^{i t}\right) \\
-\cos \frac{\phi}{2} \sin \frac{\phi}{2}\left(1-e^{-i t}\right) & e^{-i t} \cos ^{2} \frac{\phi}{2}+\sin ^{2} \frac{\phi}{2}
\end{array}\right) .
$$

As seen before, this matrix is similar to $\exp \left(\omega H_{\rho}\right)$, where

$$
\omega=\cos ^{-1}\left(\sin ^{2} \frac{\phi}{2}+\cos ^{2} \frac{\phi}{2} \cos t\right) \text {. }
$$

By observation (thanks to John Cornwall for making it easier), there exists a constant $C=C_{t} \in(0,1)$ such that

$$
\cos (\pi-\phi) \leq \sin ^{2} \frac{\phi}{2}+\cos ^{2} \frac{\phi}{2} \cos t \leq \cos C(\pi-\phi) \quad \forall \phi \in\left(\pi-\frac{1}{2}, \pi\right),
$$

and accordingly

$$
0<C(\pi-\phi) \leq \omega \leq \pi-\phi<\frac{1}{2} \quad \forall \phi \in\left(\pi-\frac{1}{2}, \pi\right) .
$$

Hence we find that

$$
f\left(h_{t} k_{\phi} h_{t} k_{-\phi}\right)=f\left(\exp \left(\omega H_{\rho}\right)\right)=\frac{\omega^{-2}}{\log \omega^{-1}} \geq \frac{(\pi-\phi)^{-2}}{\log \{C(\pi-\phi)\}^{-1}},
$$

for all $\phi \in\left(\pi-\frac{1}{2}, \pi\right)$. It therefore follows that

$$
\begin{aligned}
\mathscr{M} f(x) & \geq \frac{1}{2} \int_{\pi-1 / 2}^{\pi} \frac{(\pi-\phi)^{-2}}{\log \{C(\pi-\phi)\}^{-1}} \sin \phi d \phi \\
& \geq \frac{1}{4} \int_{\pi-1 / 2}^{\pi} \frac{(\pi-\phi)^{-1}}{\log \{C(\pi-\phi)\}^{-1}} d \phi \\
& =\frac{1}{4} \int_{0}^{C / 2} \frac{\varphi^{-1}}{\log \varphi^{-1}} d \varphi=\frac{1}{4} \int_{\log (2 / C)}^{\infty} \frac{d \psi}{\psi}=\infty,
\end{aligned}
$$

as claimed.

\section{REFERENCES}

[1] M. Christ, Averages of functions over submanifolds, preprint.

[2] M. Cowling and C. Meaney, On a maximal function on compact Lie groups, Trans. Amer. Math. Soc., 315 (1989), 811-822.

[3] J. E. Humphreys, Introduction to Lie Algebras and Representation Theory, Springer-Verlag, New York, 1972.

[4] A. A. Sagle and R. E. Walde, Introduction to Lie Groups and Lie Algebras, Academic Press, New York, 1973.

[5] C. D. Sogge and E. M. Stein, Averages over hypersurfaces III-Smoothness of generalized Radon transforms, preprint.

[6] E. M. Stein, Maximal functions: spherical means, Proc. Nat. Acad. Sci. U.S.A., 73 (1976), 2174-2175. 
[7] V. S. Varadarajan, Lie Groups, Lie Algebras, and Their Representations, Prentice-Hall, Englewood Cliffs, N.J., 1974.

[8] N. R. Wallach, Harmonic Analysis on Homogeneous Spaces, Marcel-Dekker, New York, 1973.

[9] D. P. Želobenko, Compact Lie Groups and Their Representations, Amer. Math. Soc., Providence, RI, 1973.

Received March 21, 1991 and in revised form October 16, 1991.

The University of New South Wales

KENSINGTON, N.S.W. 2033

Australia

Correspondence address: Jurusan Matematika

Institut Teknologi Bandung

Jalan Ganesha 10 Bandung

Indonesia 


\title{
PACIFIC JOURNAL OF MATHEMATICS
}

Founded by

\author{
E. F. BECKENBACH (1906-1982) F. WolF (1904-1989) \\ EDITORS
}

\author{
V. S. VARADARAJAN \\ (Managing Editor) \\ University of California \\ Los Angeles, CA 90024-1555 \\ vsv@math.ucla.edu \\ Herbert Clemens \\ University of Utah \\ Salt Lake City, UT 84112 \\ clemens@math.utah.edu \\ F. Michael Christ \\ University of California \\ Los Angeles, CA 90024-1555 \\ christ@math.ucla.edu \\ ThOMAs ENRIGHT \\ University of California, San Diego \\ La Jolla, CA 92093 \\ tenright@ucsd.edu
}

\author{
Nicholas ERCOLANI \\ University of Arizona \\ Tucson, AZ 85721 \\ ercolani@math.arizona.edu \\ R. FINN \\ Stanford University \\ Stanford, CA 94305 \\ finn@gauss.stanford.edu \\ VAUGHAN F. R. JONES \\ University of California \\ Berkeley, CA 94720 \\ vfr@math.berkeley.edu \\ Steven KerckhofF \\ Stanford University \\ Stanford, CA 94305 \\ spk@gauss.stanford.edu
}

C. C. Moore

University of California

Berkeley, CA 94720

MaRTin SCHARLEMANN

University of California

Santa Barbara, CA 93106

mgscharl@henri.ucsb.edu

HAROLD STARK

University of California, San Diego

La Jolla, CA 92093

\author{
UNIVERSITY OF ARIZONA \\ UNIVERSITY OF BRITISH COLUMBIA \\ CALIFORNIA INSTITUTE OF TECHNOLOGY \\ UNIVERSITY OF CALIFORNIA \\ MONTANA STATE UNIVERSITY \\ UNIVERSITY OF NEVADA, RENO \\ NEW MEXICO STATE UNIVERSITY \\ OREGON STATE UNIVERSITY
}

\section{SUPPORTING INSTITUTIONS}

UNIVERSITY OF OREGON

UNIVERSITY OF SOUTHERN CALIFORNIA

STANFORD UNIVERSITY

UNIVERSITY OF HAWAII

UNIVERSITY OF TOKYO

UNIVERSITY OF UTAH

WASHINGTON STATE UNIVERSITY

UNIVERSITY OF WASHINGTON 


\section{PACIFIC JOURNAL OF MATHEMATICS}

Volume $156 \quad$ No. $1 \quad$ November 1992

Enveloping algebras of Lie groups with discrete series

Nguyen HuU AnH and VuOng Manh Son

Asymptotic behavior of eigenvalues for a class of pseudodifferential operators on $\mathbf{R}^{n}$

JUNICHI ARAMAKI

A hybrid of theorems of Vinogradov and Piatetski-Shapiro

ANTAL BALOG and JOHN BENJAMIN FRIEDLANDER

Chaos in terms of the map $x \rightarrow \omega(x, f)$

ANDREW MiCHAEL BRUCKNER and JACK GARY CEDER

Local real analytic boundary regularity of an integral solution operator 97 of the $\bar{\partial}$-equation on convex domains

ZHENHUA CHEN

On some properties of exhaustion maps between bounded domains

Chi Keung Cheung

A generalization of maximal functions on compact semisimple Lie groups

HENDRA GUNAWAN

Stability of nonsingular group orbits

CLARK DEAN HORTON

Bordism and regular homotopy of low-dimensional immersions

JOHN FORBES HuGHES

On six-connected finite $H$-spaces

JAMES PEICHENG LiN and Frank WiLliams 